\title{
The Role of Iron Staining in Myelodysplastic Syndromes: A Treasure Trove of Information
}

\author{
Giacomo Marchi Fabiana Busti Domenico Girelli
}

Internal Medicine, University Hospital of Verona, Verona, Italy

Iron metabolism is tightly linked to erythropoiesis in health and disease. Almost all circulating iron in blood is destined for erythropoietic need in a strictly regulated process. Erythroferrone, whose expression directly correlates with the erythropoietic demand, is emerging as the main erythroid regulator of iron metabolism. Its production suppresses hepcidin, the master regulator of iron absorption, circulation, and distribution. An altered iron metabolism is a well-known feature of myelodysplastic syndromes (MDS), resulting into systemic iron overload, which in turn may represent the main clinical issue determining morbidity and mortality in low-risk MDS, even in non-transfusion-dependent patients. Refractory anemia with ringed sideroblasts (RARS) represents the prototype of low-risk MDS associated with iron overload. It is usually associated with somatic mutations in the SF3B1 gene, encoding for a splicing factor, and is characterized by erythroid dysplasia, anemia, $<5 \%$ of blasts, and $\geq 15 \%$ of ringed sideroblasts in the bone marrow as detected by the Prussian blue staining (also known as Perls' staining). Iron overload in RARS is linked to low hepcidin levels. Indeed, the lowest hepcidin levels have been detected in RARS compared to other MDS, even adjusting for transfusion history [1]. The loss of hepcidin regulation causes an increased iron absorption and recycling leading to tis-

karger@karger.com

(c) 2020 S. Karger AG, Basel

www.karger.com/aha

Karger sue iron overload, especially in the liver and heart. However, the dysregulation of iron homeostasis may also have a local impact in the bone marrow. Although the toxic effects of free iron through the Fenton reaction are well known, their detrimental effects in the erythropoietic niche and on the proliferation and maturation of hematopoietic stem cells have been highlighted only recently.

In this issue of the journal, Pilo and colleagues investigated the predictive value of stainable bone marrow iron on overall survival (OS) in 83 low-IPSS risk and 31 intermediate-I IPSS risk MDS patients [2]. They found that the majority of patients had grade 2 or 3 Perls' staining of bone marrow aspirate (27 and 49\%, respectively), and that OS was significantly lower in these subgroups, nearly halved as compared to Perls grade 1 group. The causes of death in the Perls grade 3 group were infections or leukemia in $55.5 \%$ of cases, and these patients had lower hemoglobin at diagnosis and were more frequently transfusion-dependent [2].

These findings substantially complement those of a recent meta-analysis, which showed that iron chelation therapy (ICT) can ameliorate anemia, and results into a lower risk of leukemia transformation and mortality in MDS patients [3]. On the other hand, other studies have suggested that iron can drive tumor cells killing. Indeed, 
heme and iron can shift the polarization of tumor-associated macrophages into an M1 pro-inflammatory phenotype producing reactive oxygen species and pro-inflammatory cytokines, which in turn cooperate in killing tumor cells [4]. In MDS patients, a positive correlation between infiltration of M1-tumor-associated macrophages and OS has been observed [5]. These apparently opposite observations are likely the two faces of the same medal, showing that iron metabolism has to be tightly regulated in the bone marrow, avoiding both functional iron deficiency and overload. Further studies are needed to better elucidate the pathogenic role of iron in MDS complications, as well as to establish novel therapeutic strategies in order to avoid detrimental effects on hematopoietic stem cells.

\section{Conflict of Interest Statement}

The authors have no conflicts of interest to declare.

\section{Funding Sources}

No funding sources were used for this manuscript.

\section{Author Contributions}

G.M. wrote the manuscript, F.B. co-wrote the manuscript, and D.G. critically revised the manuscript.

\section{References}

1 Santini V, Girelli D, Sanna A, Martinelli N, Duca L, Campostrini N, et al. Hepcidin levels and their determinants in different types of myelodysplastic syndromes. PloS One. 2011 Aug;6(8):e23109.

2 Pilo F, Caocci G, Mele G, La Nasa G. The Perls stain grade in the bone marrow aspirate correlates with overall survival in low risk myelodysplastic patients. Acta Haematologica. 10.1159/000510111.

3 Liu H, Yang N, Meng S, Zhang Y, Zhang H, Zhang W. Iron chelation therapy for myelo- dysplastic syndrome: a systematic review and meta-analysis. Clin Exp Med. 2020 Feb;20(1): $1-9$.

4 Costa da Silva M, Breckwoldt MO, Vinchi F, Correia MP, Stojanovic A, Thielmann CM, et al. Iron induces anti-tumor activity in tumorassociated macrophages. Front Immunol. 2017 Nov; 8:1479.

5 Yang $\mathrm{Y}, \mathrm{Wu} \mathrm{Y}$. The clinical significance of tumor associated macrophages in myelodysplastic syndromes. Blood. 2018 Nov; 132(1): 5505 . 